\title{
Talker and accent familiarity yield advantages for voice identity perception: a voice sorting study
}

\author{
Sheriff Njie $^{1 *}$, Nadine Lavan ${ }^{1,2^{*}} \&$ Carolyn McGettigan ${ }^{1}$ \\ *These authors contributed equally
}

${ }^{1}$ Department of Speech, Hearing and Phonetic Sciences, University College London

${ }^{2}$ Department of Psychology, School of Biological and Chemical Sciences

Queen Mary University of London

Correspondence to:

Nadine Lavan, Department of Psychology, School of Biological and Chemical Sciences

Queen Mary University of London, Mile End Road, London E1 4NS, United Kingdom.

E-mail: n.lavan@qmul.ac.uk

or

Carolyn McGettigan, Department of Speech, Hearing and Phonetics Sciences, University College London, Chandler House 2 Wakefield Street, London WC1N 1PF, United Kingdom

E-email: c.mcgettigan@ucl.ac.uk

Funding: This work was supported by a Research Leadership Award from the Leverhulme Trust (RL-2016-013) awarded to Carolyn McGettigan and a Sir Henry Wellcome Fellowship (220448/Z/20/Z) awarded to Nadine Lavan 
Talker and accent familiarity yield advantages for voice identity perception

\begin{abstract}
Familiarity benefits in voice identity perception have been frequently described in the literature. Typically, studies have contrasted listeners who were either familiar or unfamiliar with the target voices, thus manipulating talker familiarity. In these studies, familiarity with a voice results in more accurate voice identity perception. Such talker familiarity is, however, only one way in which listeners can be familiar with the stimuli used: Another type of familiarity that has been shown to benefit voice identity perception is language or accent familiarity. In the current study, we examine and compare the effects of talker and accent familiarity in the context of a voice identity sorting task, using naturally varying voice recording samples from the TV show "Derry Girls". Voice samples were thus all spoken with a regional accent of UK/Irish English (Northern Irish). We tested four listeners groups: Listeners were either familiar or unfamiliar with the TV show (and therefore the talker identities) and were either highly familiar or relatively less familiar with the accent. We find that both talker and accent familiarity significantly improve accuracy of voice identity perception. However, the effect sizes for effects of talker familiarity are overall larger. We discuss our findings in light of existing models of voice perception, arguing that they provide evidence for interactions of speech and identity processing pathways in voice perception. We conclude that voice perception is a highly interactive process, during which listeners make use of any available information to achieve their perceptual goals.
\end{abstract}

Keywords: voice identity, accent, familiarity, within-person variability 
Talker and accent familiarity yield advantages for voice identity perception

\title{
Introduction
}

\author{
Orla: "Why's he making that funny noise?" \\ Michelle: "He's English Orla, that's the way they talk."
}

Derry Girls

Human listeners can perceive identity-related information from voices (Kreiman \& Sidtis, 2011; Mathias \& Von Kriegstein, 2014). How accurately listeners can perform voice identity perception tasks depends on many factors related to the properties of the stimuli used, and the characteristics of the listeners. In terms of listener characteristics affecting voice identity perception, it has been reported that, broadly speaking, familiarity with aspects of the stimuli used is advantageous.

Effects of familiarity on voice identity perception have most frequently been tested by contrasting listeners that are either familiar or unfamiliar with the voices used (Lavan, Kreitewolf et al., 2020; Lavan, Burston \& Garrido, 2019; Lavan, Burston, Ladwa et al., 2019; Lavan, Merriman et al., 2019; Lavan et al., 2016; Stevenage et al., 2020). These studies typically use voice identity discrimination or voice identity sorting paradigms, allowing familiar and unfamiliar listeners' performance to be compared directly on the same task. They have furthermore focused on the effects of talker familiarity in the context of voice stimuli that include within-talker variability. Within-talker variability describes the observation that the sound of a single person's voice can change dramatically, depending on the speaking situation: People change the sound of their voice to best convey their intentions and feelings, and to adapt to their audience and acoustic environment (Lavan, Burton et al., 2019). The results of this body of work on talker familiarity effects have converged on two key findings: 1) within-talker variability generally presents challenges to voice identity perception but 2) being familiar with a talker enables listeners to largely overcome these challenges, resulting in more accurate identity perception.

Specifically, in voice identity sorting studies, listeners who are either familiar or unfamiliar with the talkers are presented with a limited number of naturally-varying voice recordings embedded on a drag-and-drop interface (e.g. Lavan, Burston \& Garrido, 2019; Stevenage et al., 2020). Such naturally-varying voice recordings include different speaking situations, across which properties such as the emotional content (e.g. happy vs angry) or the speaking style (e.g. formal vs casual; high vs low effort) vary naturally. The voice recordings used are usually sampled from 2-3 identities, represented by a number of recordings each. Listeners are then asked to listen to the voice recordings and sort them into clusters by identity. The pattern of results that emerges across all studies is that listeners who are not familiar with the talkers perceive many more identities than are actually present, as indicated by a larger number of clusters. When examining how these different clusters are formed, it becomes apparent that the unfamiliar listeners perceive variable voice recordings from the same talker as multiple different talkers. Unfamiliar listeners thus frequently fail to "tell together" variable voice recordings, misinterpreting within-talker variability as between-talker variability. Crucially, if listeners are familiar with the talkers, accuracy improves drastically: While performance is not perfect, familiar listeners tend to 
accurately recognise the within-talker variability as such, and therefore perceive variable examples of a talker's voice as the same identity. Speaker discrimination studies contrasting familiar and unfamiliar listeners furthermore corroborate these benefits of being familiar with the talkers (Lavan \& Kreitewolf et al., 2020; Lavan et al., 2016).

Familiarity with the talkers in a task is, however, only one type of familiarity that may benefit listeners when making identity judgements. Other studies have shown that being familiar with the language used by the talkers producing the stimuli can result in more accurate identity perception, a finding referred to as the language-familiarity effect (Perrachione, 2017 for a recent review): Studies using a variety of voice identity discrimination, line-up, or recognition tasks consistently report better performance when the language used in the voice recordings is the listeners' native language. Zarate et al. (2015) furthermore report that language familiarity advantages appear to work along a gradient, where advantages can arise across different languages, if these languages share common phonological information.

Advantages in identity perception due to accents have also been reported: Goggin et al. (1991) report that for monolingual English speaker, voice identity perception is less accurate for Spanish-accented English compared to American-accented English. A number of studies have furthermore investigated whether familiarity with a regional accent within a listeners' native language may also aid identity perception. A study focussing on forensic voice identification (Stevenage, et al., 2012) reports that voice identification is indeed more accurate in a voice line-up when the talker is speaking with the listeners' own accent (English vs Scottish). Kerstholt et al. (2006) furthermore report similar effects of more accurate voice-line up identification for a familiar (standard) Dutch accent versus a less familiar regionally and socially marked accent from The Hague. Braun et al. (2018) even report some accent familiarity advantages at a sub-regional level for voice line-ups, contrasting different accents from the NorthEast of England (Newcastle, Sunderland and Middlesbrough). In this study, misses (that is, discounting the target speaker as a foil voice) were significantly lower in the listeners' own specific accent. Performance for hits and false alarms was, however, comparable. However, Johnson, et al. (2018) report no own-accent advantage in a talker discrimination task when contrasting American and Australian English. Beyond regional accents, socio-cultural accents (e.g., Black American English vs White American English) can elicit own-accent advantages (Perrachione et al., 2010). Thus, there is largely consistent evidence for a language familiarity effect when contrasting voice identity perception in the listeners' native vs a foreign language, as well as ownaccent (defined primarily via regional or socio-cultural criteria) advantages across different experimental tasks.

Several explanations have been proposed for when and why both talker familiarity and language or accent familiarity advantages arise for voice identity perception. For identity perception, it has been proposed that familiar listeners can access a talkerspecific representation of the voices in question. This representation is likely to include information about how the voice of a familiar identity can vary (Lavan, Burton et al., 2019). Such a representation may then enable familiar listeners to cope with the 
within-talker variability of familiar voices and arrive at an accurate identity percept. Unfamiliar listeners, however, lack such a talker-specific representation and thus must rely on more general knowledge of how voices can vary when deciding whether two variable recordings of voices were produced by the same speaker or two different speakers (Lavan, Burston et al., 2019; Lavan et al., 2016). For language familiarity benefits, the key explanations ascribe benefits to primarily being familiar with the acoustic-phonetic features of the language (see Perrachione, 2017 for a recent review). An alternative model suggests that the ability to process the linguistic information in the stimuli - beyond its acoustic-phonetic properties - is additionally contributing to familiarity benefits in voice identity perception. What these explanations have in common is that they share the proposal that familiarity enables listeners to process linguistic information that unfamiliar listeners cannot process effectively. Familiarity with a talker allows the listener to access information about the possible variability in the sound of a specific talker, while language or accent familiarity allows the listener to - potentially additionally - perceive idiosyncrasies in the speech of a talker (e.g., a characteristic way of pronouncing a certain vowel) that would not be salient to an unfamiliar listener. Intriguingly, the reported advantages for accent and language familiarity during identity processing, alongside the mechanistic explanations, are partially at odds with a classic model of voice perception (Belin et al., 2004). In that model, the processing of speech- and identity-related information can under certain circumstances interact, but are in the end considered to be largely independent of one another.

To our knowledge, talker-familiarity and language-familiarity related benefits during voice identity perception have so far only been investigated in isolation. Similarly, studies of accent familiarity have to date not considered naturally-varying stimuli in their tasks. In the current study, we therefore investigated effects of familiarity with both talkers and their accent in a voice identity sorting task. We used naturally-varying voice recordings from two characters from the TV show "Derry Girls". Stimuli in our study thus feature a pronounced Northern Irish accent. We then tested 4 groups of listeners: To manipulate talker-familiarity, we recruited listeners who were either familiar or unfamiliar with the TV show (and thus the identities). To manipulate accentfamiliarity, we recruited listeners who were either from Northern Ireland (and thus highly familiar with the accent) or from the East of England (and thus less familiar with the accent). We note at this stage that in the experimental design described above, talker and accent familiarity are partially overlapping. For example, listeners from the East of England who have watched the TV show "Derry Girls" have become familiar with the different talkers - but at the same time, they will also have become more familiar with the Northern Irish accent. Our results therefore need to be interpreted in light of these partially overlapping types of familiarity.

Based on previous research on the effect of familiarity with the talker, we predicted that listeners who are familiar with the talkers will show significantly higher accuracy in voice perception than listeners who are not familiar with the voices. We furthermore predicted that accent familiarity would have significant effects on identity perception, such that accuracy would be higher for listeners who are familiar with the accent. Additionally, we predicted that the effects of accent familiarity may be smaller than the effects of talker familiarity, on the basis that speech perception (accent familiarity) is 
Talker and accent familiarity yield advantages for voice identity perception

less directly related to the task of talker identification than knowledge of the talkers themselves (talker familiarity).

\section{Methods}

\section{Participants}

In total 165 participants were tested for this study. All participants were aged between 18-40 years, were native speakers of English, and did not have any self-reported hearing difficulties or language processing difficulties. We recruited four groups of participants via the online recruitment platform Prolific.co: The groups included participants who were either familiar or unfamiliar with the TV show "Derry Girls" and participants who were either highly familiar with Northern Irish accents or less familiar with Northern Irish accents.

Familiarity with Northern Irish accents was primarily established using preselection criteria in Prolific: Using these criteria, familiar participants were defined as those who had spent the first 18 years of their lives mostly in Northern Ireland and were currently still living there. Conversely, participants who were deemed to be less familiar with the Northern Irish accent were recruited based on having spent most of the first 18 years of their lives in England and currently living in either the East or South East of England. As a secondary assessment, accent familiarity was assessed via self-report by asking participants to rate their familiarity with a range of UK regional accents (e.g. Welsh, Cockney, Northern Irish, etc.) on a scale form 1 (not familiar at all) to 10 (very familiar). A range of accents was included to reduce the prominence of the rating on the Northern Irish accent in this questionnaire, although it was our only measure of interest. Familiarity with the TV show was established via self-report at the outset of the task (see Methods).

From the sample, 8 participants were excluded because they failed to accurately complete a vigilance check (sorting 2 identical recordings of a computer-generated male voice into an independent cluster; see Materials and Procedure). Nine further participants were excluded due to having some pre-existing familiarity with the talkers - these participants reported to not have watched the TV show but in the debrief questionnaire (see Procedure) reported to have recognised one or both talkers from other TV shows or media coverage. Nineteen participants who had reported having watched the TV show "Derry Girls" were excluded because they reported having watched less than one season of the show, and as such were considered to be insufficiently familiar with the characters. Finally, 3 participants were excluded since they formed only one cluster after spending a short amount of time on the sorting task - this pattern of responses had not been observed in any of the previous identity sorting tasks run in our research group and was thus considered to be sufficiently anomalous to warrant the exclusion of these participants.

After these exclusions, data from 126 participants remained. There were 32 participants in the group that was familiar with both the talkers and the accent (Mean age $=25.5$ years, $S D=5.9 ; 24$ female), 29 participants in the group that was familiar with the talker but less familiar with the accent (Mean age $=28.1$ years, $S D=5.9 ; 14$ 
female), 32 participants in the group that was familiar with the accent but unfamiliar with the talker (Mean age $=27.2$ years, $S D=4.8 ; 11$ female), and 33 participants group that was not familiar with either the talkers nor the accent (Mean age $=27.6$ years, $S D=6.1,24$ female). Participants in the groups that were less familiar with the Northern Irish accent on average rated their familiarity with Northern Irish accents at 3.66 out of 10 (2.70 for listeners unfamiliar with the talkers vs 4.79 for listeners familiar with the talkers). Participants from Northern Ireland rated their familiarity on average at 9.63 out of 10 (9.34 for listeners unfamiliar with the talkers vs 9.91 for listeners familiar with the talkers). The study was run 2.5 years after the first season of the TV show was first broadcast, and just over 1 year after the second season had been first broadcast.

\section{Materials}

In the current voice sorting task, we used 15 naturally-varying voice recordings each from two characters from the TV "Derry Girls". The TV show "Derry Girls" was chosen for the purpose of this study as it is set in Northern Ireland, featuring the local accent of Derry. The actors playing the two characters chosen (Saoirse-Monica Jackson as Erin Quinn and Jamie-Lee O'Donnell as Michelle Mallon) are both from Derry themselves, and thus were speaking/acting in their native accent. Recordings were sampled from across different scenes, speaking situations, and speaking environments, thus varying in an unconstrained manner in their linguistic content, speaking style, emotional content, and intention conveyed, among other factors. This was done to sample as much of the natural within-person variability of each identities voice as possible. Each voice recording included a full meaningful utterance (such as "We had a few questions about the British Empire"), and included as little background noise as possible, while no other voices were audible. Recordings were furthermore selected to be non-diagnostic, avoiding catch phrases for what were considered to be particularly memorable scenes or lines. All experimental stimuli were RMS normalised and the average duration of these voice recordings was 2.74 seconds $(S D=0.58)$. We also generated a sound clip of a male voice via a text-to-speech synthesiser ("The violin is a beautiful instrument but tough to learn"), which was used in a vigilance check (see below).

These voice recordings were embedded in a PowerPoint slide, with each clip being represented by a numbered box. The numbered boxes were distributed across the slide in a random pattern with no clear clusters being apparent from the outset. The computer-generated voice was added twice to the Powerpoint slide, forming the basis of the vigilance task. Participants were expected to readily spot the two duplicate recordings of a male voice and sort them into a cluster of their own, such that these duplicate recordings were used as catch trials. If a participant did not correctly sort these two recordings into a cluster on their own they were excluded from the data analysis.

\section{Procedure}

The experiment was implemented using the Qualtrics survey software (Qualtrics, Provo, UT). Participants were first presented with an information sheet and gave their informed consent to take part in the study. Participants then stated whether they had 
watched the TV show "Derry Girls" or not. They were then asked to download the Powerpoint slide including the voice recordings. Participants were informed that this Powerpoint slide included 32 short recordings of voices, linked to numbered boxes (see Materials). They were then asked to listen to these voice recordings and sort them by perceived identity into clusters, such that each cluster only included recordings produced by the same person. Participants could play the voice recordings in whichever order they chose and could replay recordings as many times as they considered necessary. Participants were unaware that the 'true' number of identities included in the study was in fact two (plus the third, male voice used for the catch trials). Sorting was achieved by participants dragging and dropping the boxes on the Powerpoint slide. Participants were shown 3 examples that illustrated what a sorted Powerpoint slide could look like. These depicted variable numbers of clusters, and participants were told not be led by the number of clusters shown in these examples. Finally, to minimise ambiguities with regard to which sound files were included in which clusters, participants were asked to circle each cluster.

After participants had completed the sorting task to their satisfaction, they were asked to upload the sorted slide to a website supporting anonymous file sharing. All participants then completed a debrief questionnaire in which they indicate whether they recognised any of the voices present and were asked to describe their strategy for completing the task. For participants who reported to be familiar with the TV show "Derry Girls" participants were asked which seasons of "Derry Girls" they had watched ("No full season", "At least one season", "Both seasons"; see exclusions). They were also asked how many of the specific voice recordings they may have remembered from the show (with the options 0-5, 6-10 and 10+). Due to an error, data for this question was not collected for all familiar participants. However, an inspection of the data that were collected indicated that the distribution of responses was comparable for listeners who were highly vs less familiar with the accents. We note that this lack of insight into how many stimuli participants perceived to have remembered from watching the TV show potentially presents a confound. We discuss this issue at length in the Discussion section. The experiment took around 20 minutes to complete and participants were reimbursed for their time at a rate of $£ 7.50$ per hour.

\section{Data processing}

Three key task performance measures were extracted: 1) Number of clusters formed, 2) a "telling together" score, and 3) a "telling apart" score. The computer-generated voice recordings associated with the vigilance task were excluded at the stage of the data processing.

1) The number of clusters corresponds to the number of perceived talkers. With two talkers being the correct answer, worse performance is indexed by a greater number of clusters. Clusters were counted directly from the sorted Powerpoint slide.

2) The "telling together" score represents listeners' ability to accurately perceive several variable recordings of a talker as the same identity: To calculate this 
scores, we first created a response matrix of all possible pairwise combinations of the voice recordings (see Figure 1). A pair of stimuli from the same talker was coded as 1 if sorted into the same cluster (i.e. accurately "told together") or 0 if sorted into different clusters. Conversely, a pair of stimuli from different talkers was coded as 0 if sorted into different clusters ("accurately told apart") and 1 if sorted into the same cluster. The "telling together" score was then computed by taking the average of all pairs that veridically include the same talker (see also Lavan, Burston, \& Garrido, 2019). If participants have sorted all voice recordings correctly, the "telling together" score is 1. However, if participants split voice recordings from the same talker into multiple clusters, the "telling together" score decreases, indicating worse performance.

3) The "telling apart" score represents listeners' ability to accurately perceive variable recordings of different talkers as being separate identities. This score is calculated using the same coding strategy as the "telling together" score. For the "telling apart" score, however, the 1s and 0s are averaged across all pairs that veridically include the two different talkers. If participants never mix voice recordings of different talkers within the same cluster, the "telling apart" score is 0 . If participants mix voice recordings within the same clusters, the "telling apart" score increases, indicating worse performance.

Data were not normally distributed for some of the voice identity sorting performance measures across groups. We therefore used pairwise Mann-Whitney tests to assess the effects of talker and accent familiarity on all performance measures in our statistical analysis. These tests were implemented in the coin package (Hothorn, et al., 2006) in $R$. The effect size $r$ reported was computed in the rstatix package (Kassambara, 2020) in $R$. Effect sizes between 0.1 and 0.3 are considered to be small, 0.3 and 0.5 are considered as medium, and effect sizes over 0.5 are considered to be large. To test our hypothesis that effects of talker familiarity should overall be larger than effects of accents, we qualitatively compared and interpreted these effect sizes. 


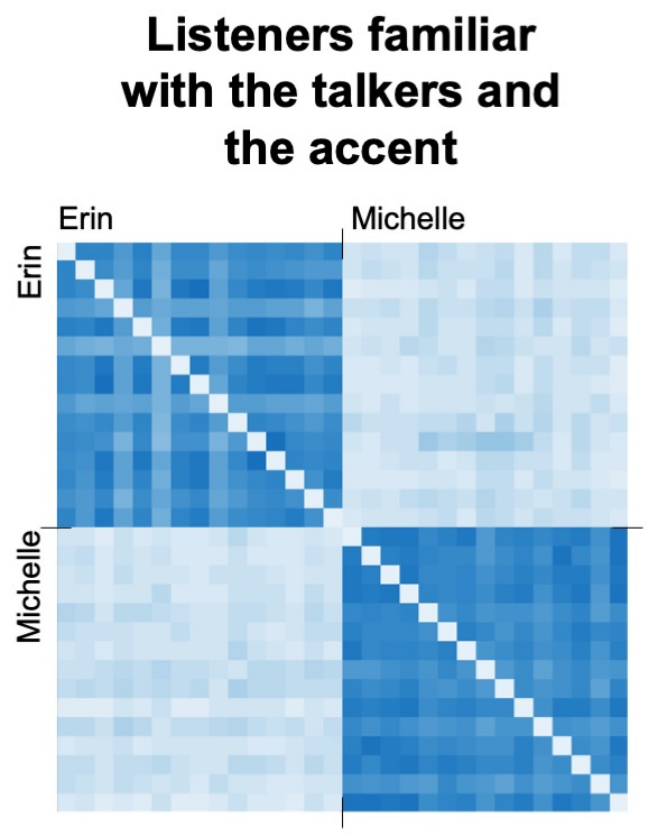

\section{Listeners familiar with the talkers and but less familiar the accent}

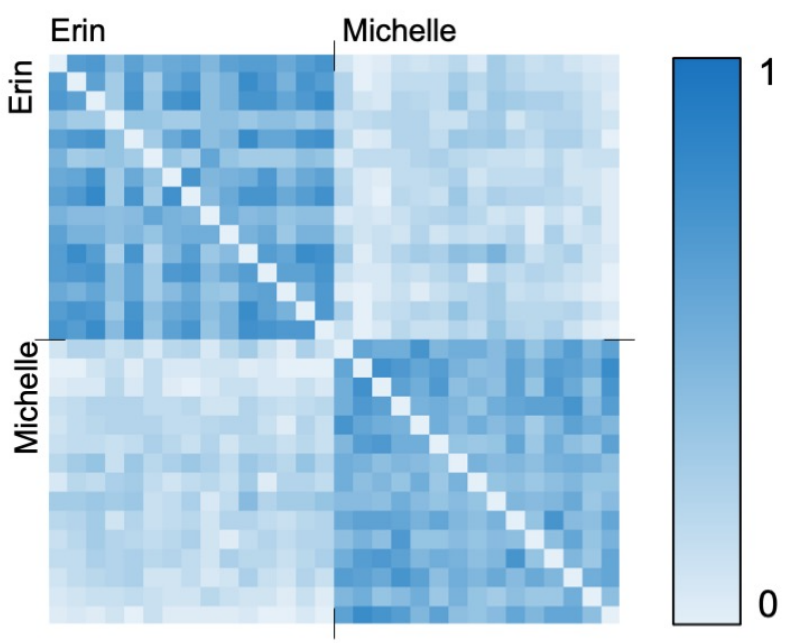

\section{Listeners familiar with the accent but not the talkers}

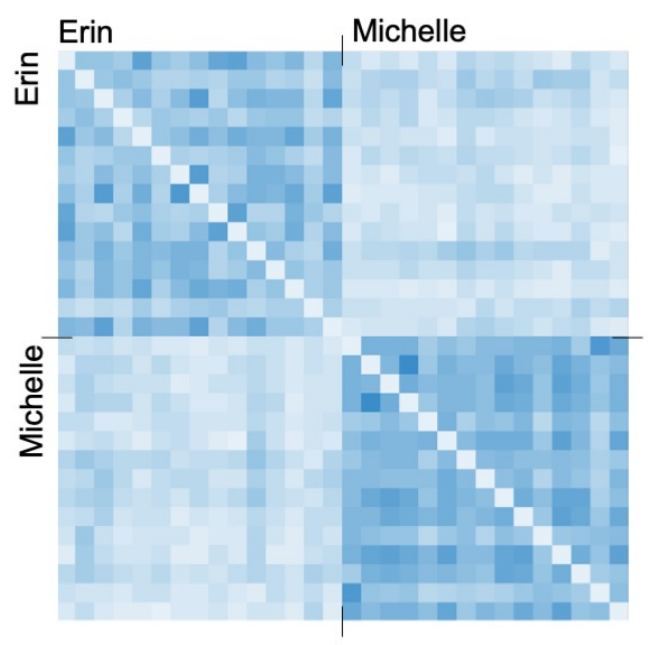

\section{Listeners unfamiliar with the talkers and the accent}

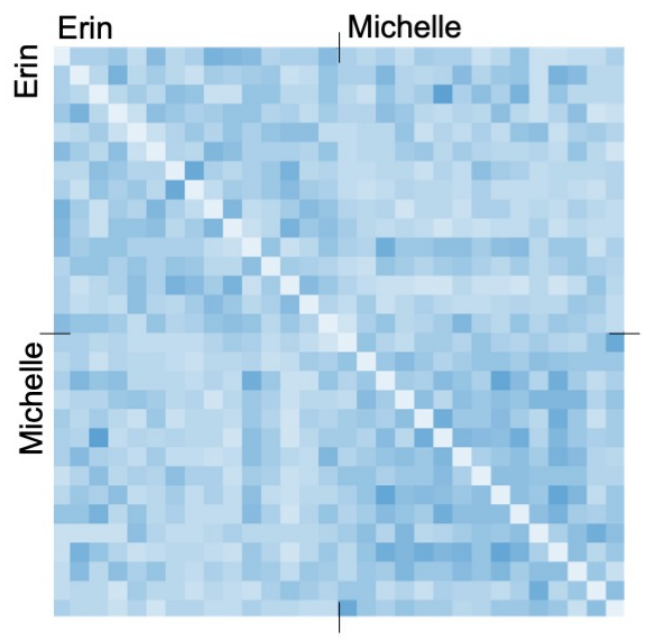

Figure 1. 30 × 30 response matrices visualising identity sorting behaviour in each of the four listener groups. Each square represents a pair of voice recordings, where the colour coding indexes how frequently this pair of identities was sorted into the same cluster in the specific listener group $(0=$ never, $1=$ always). Perfect performance would be indicated by all within-identity pairs being 1 (i.e. dark shading in the upper left-hand quarter and lower right-hand quarter of the matrix, indexing listeners' ability to "tell people together"), and all across-identity pairs being 0 (i.e. very light shading in the upper left-hand quarter and lower right-hand quarter of the matrix, indexing listeners' ability to "tell people apart"). A colour version of this figure can be found in the online version of the paper. 


\section{Results}

a)

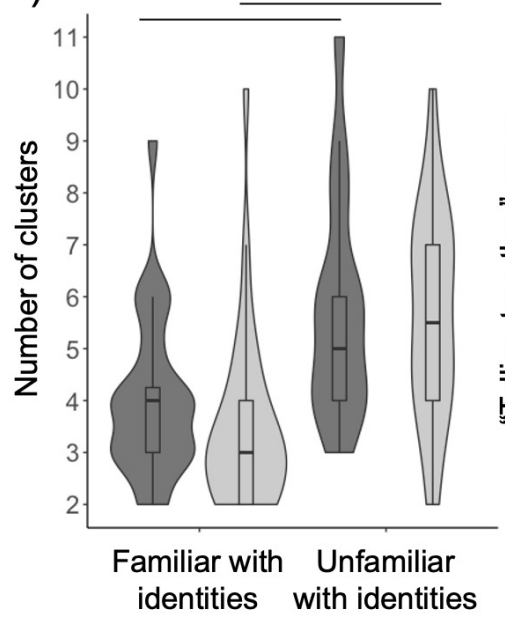

b)

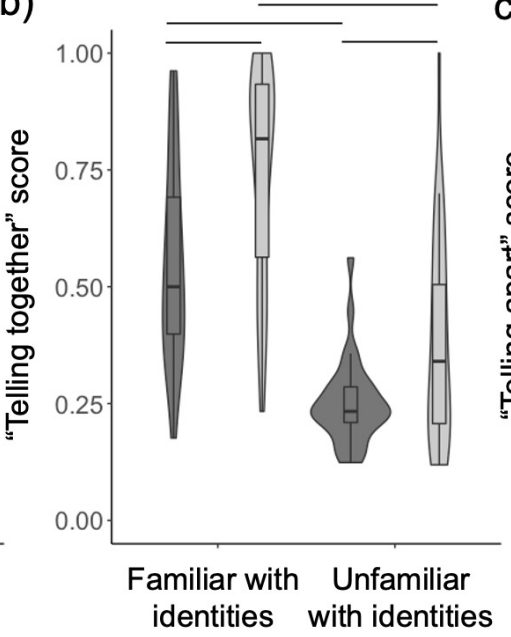

c)

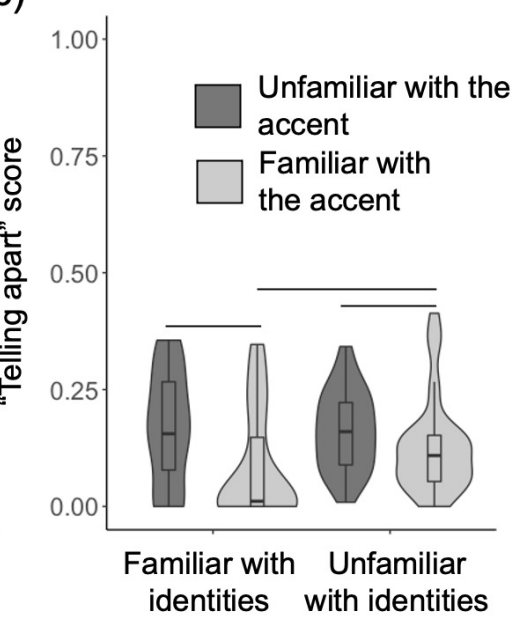

Figure 2. Performance on the voice sorting tasks, plotted by listener group. Lines above the violin plots indicate significant pairwise comparisons $(p<.05)$. For a description of how the "telling together" and "telling apart" scores were computed, see the main text and Figure 1.

\section{Effects of talker familiarity}

As reported in previous voice and face sorting studies (Jenkins et al., 2011; Lavan, Burston \& Garrido, 2019; Stevenage et al., 2019), sizeable effects of talker familiarity were apparent in our data (Figure 2).

Listeners who were familiar with the talkers through having watched the TV show formed significantly fewer clusters than listeners who were not familiar with the talkers, for both accent familiarity groups. Thus, listeners who were familiar with the talkers and the accent formed significantly fewer clusters (median number $=3$ clusters [range $=2-10]$ ) than listeners who were unfamiliar with the talkers but were familiar with the accent (median number $=5.5$ clusters [range $=2-10$ ], $W=191, p<.001, r=.55$ ). Similarly, listeners who were familiar with the talkers but not familiar with the accent also formed significantly fewer clusters (median number $=4$ clusters [range $=2-9$ ]) than listeners who were familiar with neither the talkers nor the accent (median number $=5$ clusters $[$ range $=3-11] ; W=224, p<.001, r=.45$ ).

Clear effects of talker familiarity are also apparent in the "telling together" scores: Listeners who were familiar with the talkers performed significantly better at "telling people together" than listeners who were not familiar with the talkers, again for both accent groups. For listeners who were familiar with the accent, the median "telling together" score was 0.82 (range $=.23-1.0$ ) for listeners who were familiar with the talkers and 0.34 (range $=.12-1.0$ ) for listeners who were unfamiliar with the talkers $(W=887, p<.001, r=.63)$. For listeners who were less familiar with the accent, the "telling together" score was 0.50 (range $=.18-.96$ ) for listeners who were familiar with the talkers and 0.23 (range $=.12-.56 ; W=860, p<.001, r=.74$ ) for listeners who were unfamiliar with the talkers. 
For "telling people apart", listeners who were familiar with the talkers and the accent were significantly better at "telling people apart" than who were not familiar with the talkers (median score of 0.01 [range $=0-0.35$ ] vs 0.11 [range $=0-0.41$ ]; $\mathrm{W}=316, p$ $=.008, r=.33$ ). However, the two listener groups who were less familiar with the accent performed similarly for "telling people apart" (median scores of 0.16 for both groups [ranges $=$ familiar with the talkers: 0 - 0.36, unfamiliar with the talkers: 0 - .34]; $W=471, p=.902, r=.02)$.

\section{Effects of accent familiarity}

There was no significant effect of accent familiarity on the number of clusters formed by listeners. This was the case for both the listeners who were familiar with the talkers (Median number of clusters for the group that was familiar with the accent: 3 clusters [range $=2$ - 10]; Median number of clusters for the group that was less familiar with the accent: 4 clusters [range $=2$ - 9]; $W=579, p=.077, r=.23$ ) and listeners who were unfamiliar with the talkers (Median number of clusters for the group that was familiar with the accent: 5.5 clusters [range $=2$ - 10]; Median number of clusters for the group that was less familiar with the accent: 5 clusters [range $=3-11$ ]; $W=510.5$, $p=.821, r=.03)$.

Although no accent familiarity effects emerged in the number of talkers that were perceived, performance on "telling people together" and "telling people apart" showed that there were consistent effects of accent familiarity in terms of how the clusters were formed. Listeners who were highly familiar with the accent were better at "telling people together" than listeners who were less familiar with the accent. This was the case both for listeners who were familiar with the talkers (Median "telling together" score for the group familiar with the accent: 0.82 [range $=.23-1.0$ ]; Median "telling together" score for the group less familiar with the accent: 0.50 [range $=.18-.96$ ]; $W$ $=236, p=.001, r=.41$ ) and for listeners who were unfamiliar with the talkers (Median "telling together" score for the group familiar with the accent: 0.34 [range $=.12-1.0]$; Median "telling together" score for the group less familiar with the accent: 0.23 [range $=.12-.56] ; W=362, p=.029, r=.27$ ).

A similar pattern of results emerged for "telling people apart". Listeners who were highly familiar with the accent were better at "telling people apart" than listeners who were less familiar with the accent. Similar to the profile of results for "telling people together", this was the case both for listeners who were familiar with the talkers (Median "telling apart" score for the group familiar with the accent: 0.01 [range $==0$ 0.35]; Median "telling apart" score for the group less familiar with the accent: 0.16 [range $=0$ - .36]; $W=616, p=.011, r=.33$ ) and listeners who were unfamiliar with the talkers (Median "telling apart" score for the group familiar with the accent: 0.11 [range $=0-.41]$; Median "telling apart" score for the group less familiar with the accent: 0.16 [range $=0-.34$ ]; $W=690, p=.034, r=.26$ ).

Taken together, these results show that both talker and accent familiarity affect voice identity perception in a sorting task. However, where significant effects are present, 
Talker and accent familiarity yield advantages for voice identity perception

comparison of effect sizes across the pairwise comparisons shows that the effects of talker familiarity are larger than the effects of accent familiarity.

\section{Discussion}

Our study shows that both familiarity with a talker and familiarity with an accent can benefit listeners during a voice identity perception task. Familiarity with the talkers, acquired by having watched at least one season of the TV show in which they featured, had substantial effects on voice identity perception. Specifically, listeners who were familiar with the talkers outperformed who listeners who were not familiar with the talkers by perceiving significantly fewer identities in our voice sorting task. This pattern of results was also apparent for listeners' "telling together" scores, showing that listeners who were familiar with the talkers perceived naturally-varying recordings of a talker as coming from the same identity, while listeners who were unfamiliar with the talkers tended to perceive recordings of the same talker as coming from several different identities. Finally, talker familiarity effects were also apparent for "telling apart", but only for the listeners who were familiar with the accent.

Intriguingly, accent familiarity had no effect on the number of talkers perceived: Listeners from Northern Ireland and England formed a similar number of clusters. This may indicate that number of perceived talkers (as measured by counting the number of clusters formed) may in the end be a relatively crude measure of accuracy: Each cluster - be it large or small - is treated equivalently, while the accuracy of the composition of the cluster (e.g., does a cluster include recordings from only one voice or were there mixing errors?) is also not taken into account. This lack of sensitivity may therefore have obscured the effects of accent. However, when looking at potentially more sensitive measures, indexing how the different clusters were composed, consistent accent familiarity effects emerged. Compared with listeners from the East of England, listeners from Northern Ireland, who were highly familiar with the accent used in the stimuli, were better able to accurately sort multiple recordings of the same identity into the same cluster ("telling together"), while also making fewer "telling apart" errors (i.e. mixing talkers within clusters).

Our results generally support our prediction that both accent and talker familiarity can result in more accurate voice identity perception across a range of measures although we note that not all pairwise comparisons were significant. The results thus broadly align with previous reports of advantages of talker familiarity (Lavan \& Kreitewolf et al., 2019; Lavan, Burston \& Garrido, 2019; Lavan et al., 2016; Stevenage et al., 2019) and accent familiarity (Braun et al., 2018; Stevenage et al., 2011).

We had additionally predicted that effects of talker familiarity would be larger than effects of accent familiarity. Looking at the effect sizes, this indeed is indeed the case: Effects for contrasts of talker familiarity were moderate to large, while effects for contrasts of accent familiarity were small to moderate. These differences in the size of familiarity effects makes intuitive sense: Information about what a person sounds like is directly relevant to making identity judgements. In this case, talker familiarity may for example also trigger the explicit recognition of the person when hearing recording 
of their voice. Although accent familiarity can nonetheless improve accuracy for voice identity perception, accent familiarity alone can never trigger person recognition and is not directly linked to identity judgements. Overall, our results suggest that while different types of familiarity might improve the accuracy of voice identity perception, familiarity with the talkers themselves is more influential, due to the direct relationship through which this type of familiarity can inform performance on an identity perception task.

We note a caveat regarding this finding of differences in effect size. One potential limitation in the current design is that no participants were excluded (due to data collection errors) based on their ability to remember the specific recordings used in the task. As a result, "talker familiarity" may have been partially confounded with "stimulus familiarity", which may have in turn boosted the talker familiarity advantage. However, we note that the size of the effects of talker familiarity reported in this study are broadly in line with other voice identity sorting studies measuring talker familiarity effects (e.g. Lavan, Burston \& Garrido, 2019; Lavan, Burston et al. 2019; Stevenage et al., 2020). While more work is needed to replicate this finding, we thus tentatively conclude that based on the data presented in the paper as well as comparable voice identity sorting studies exploring talker familiarity, there is some evidence that effects of talker familiarity are larger than effects of accent familiarity.

Descriptively speaking, accent familiarity effects also seemed to be more pronounced for the groups of listeners who were familiar with the talkers. We did not set out to test for an interactive effect in this study, and as noted above the definition of participant groups does not allow for a clean orthogonality of the 2 familiarity types. Furthermore, we could not explicitly examine an interaction effect statistically due to the highly nonnormal nature of sorting task data, which should be analysed using non-parametric approaches. However, the patterns we have observed do suggest an intriguing and somewhat counterintuitive finding: As has been noted before, in our sample, accent familiarity is not evenly matched across groups less familiar with the accent: While both listener groups from Northern Ireland reported similarly high familiarity with the Northern Irish accent, self-reported familiarity differed between the two listener groups from the East of England (2.70/10 for listeners who had not watched the show vs $4.79 / 10$ for listeners who had watched the show). This cannot be easily avoided in this experimental design, since listeners who are familiar with the talkers from the TV show "Derry Girls" had been - by definition - actively engaged with listening to numerous Northern-Irish-accented characters who featured in the show. As a result, the difference in self-reported accent familiarity was less pronounced for comparisons of listener groups who were also familiar with the specific talkers. In this context, we might have expected the accent familiarity effect for listeners who have watched "Derry Girls" to be smaller than for unfamiliar listeners, due to the smaller difference in selfreported accent familiarity amongst those with greater talker familiarity. This is, however, not the case and the size of the accent familiarity effect was more pronounced for the listener groups who had watched the show "Derry Girls".

This difference in effect size could suggest that accent and talker familiarity are not only additive but may also interact during identity perception. For example, in line with 
mechanistic explanations for language familiarity effects (see Perrachione, 2017), listeners who were familiar with the accent could have had access to acoustic-phonetic and/or linguistic information when completing the task that listeners who were less familiar with the accent could not readily perceive. This additional information may have enabled them to also better access talker identity-related cues, improving their performance. Similarly, this potential interaction - and in fact all accent familiarity advantages reported here - may have arisen at the encoding stage, when listeners who were highly familiar with the accent watched the TV show and were already better able to encode the verbal content than listeners who were less familiar with the accent. Our study, was, however, not explicitly designed to explore such interactions or indeed to test the mechanisms through which accent familiarity advantages may arise. As such, further work designed to tackle these questions will be required.

Our findings that familiarity with aspects of the stimuli that are not directly task-relevant can improve task accuracy aligns with findings from other fields of study. For example, it has been shown that speech intelligibility in challenging listening situations is improved when a person is familiar with the talker (Kreitewolf et al., 2017; Johnsrude et al., 2013; Nygaard \& Pisoni, 1998). In the context of speech perception, familiarity with the language being spoken is clearly more crucial than any benefits that might be gained from talker familiarity. But when listening for comprehension in one's native language, it is unclear how the types of accent and identity familiarity investigated in our voice sorting study might compare. While less familiar regional accents can be difficult to understand (Stringer \& Iverson, 2019), there also differences in the intelligibility of individual talkers. Familiarity with the accent or talker may well enable listeners to cope with these listening challenges and improve speech comprehension. The size of the effects of accent and talker familiarity for speech comprehension would, however, likely depend on which information is more relevant to speech comprehension and may be strongly mediated by the specific stimuli selected.

Models of voice processing have proposed that the different processing pathways for speech, identity and emotion are largely independent - with some scope for interactions (Belin et al., 2004; Belin et al., 2011). Our results provide evidence that there are indeed many routes to achieving accurate identity perception from voices. Alongside evidence that familiarity with specific talkers can improve identity perception (Maguinness et al., 2017), we report evidence that speech-related cues can also, depending on their accessibility to the listener, provide information to support identity processing. It is perhaps unsurprising that such interactions exist between the different processing pathways (speech, identity, emotion): For example, information that is diagnostic for the identity of a talker is not only encoded in the sound of their voice but also in how they speak - including cues that vary due to societal or geographic factors alongside entirely idiosyncratic cues. In naturalistic settings, the point at which speech perception ends and voice perception begins is therefore impossible to determine.

We therefore argue that our study shows that voice processing - whether with a focus on understanding the content of speech, interpreting the emotional content, or processing person-related information - is a highly dynamic and possibly interactive process. During voice processing listeners are likely to use any information available 
Talker and accent familiarity yield advantages for voice identity perception

to them to advance their perceptual goals, irrespective of which processing pathway it may primarily belong to (Kreiman \& Sidtis, 2011, Lavan \& McGettigan, 2019). Future work is required to more fully explore when and how different kinds of information are retrieved and combined to support aspects of voice processing.

\section{Open Practices Statement}

Data are available from the authors upon reasonable requests. None of the experiments were preregistered.

\section{References}

Belin, P., Bestelmeyer, P. E., Latinus, M., \& Watson, R. (2011). Understanding voice perception. British Journal of Psychology, 102(4), 711-725.

Belin, P., Fecteau, S., \& Bedard, C. (2004). Thinking the voice: neural correlates of voice perception. Trends in cognitive sciences, 8(3), 129-135.

Braun, A., Llamas, C., Watt, D., French, J. P., \& Robertson, D. (2018). Sub-regional 'other-accent'effects on lay listeners' speaker identification abilities: a voice line-up study with speakers and listeners from the North East of England. International Journal of Speech, Language and the Law, 231-255.

Fecher, N., \& Johnson, E. K. (2018). The native-language benefit for talker identification is robust in 7.5-month-old infants. Journal of Experimental Psychology: Learning, Memory, and Cognition, 44(12), 1911-1920.

Goggin, J. P., Thompson, C. P., Strube, G., \& Simental, L. R. (1991). The role of language familiarity in voice identification. Memory \& cognition, 19(5), 448-458.

Hothorn T, Hornik K, van de Wiel MA, Zeileis A (2006). "A Lego system for conditional inference." The American Statistician, 60(3), 257-263.

Johnson, E. K., Bruggeman, L., \& Cutler, A. (2018). Abstraction and the (misnamed) language familiarity effect. Cognitive Science, 42(2), 633-645.

Johnsrude, I. S., Mackey, A., Hakyemez, H., Alexander, E., Trang, H. P., \& Carlyon, R. P. (2013). Swinging at a cocktail party: Voice familiarity aids speech perception in the presence of a competing voice. Psychological Science, 24(10), 1995-2004.

Kassambara, A. (2020). Rstatix: Pipe-Friendly Framework for Basic Statistical Tests.

Kerstholt, J. H., Jansen, N. J., Van Amelsvoort, A. G., \& Broeders, A. P. A. (2006).

Earwitnesses: Effects of accent, retention and telephone. Applied Cognitive

Psychology: The Official Journal of the Society for Applied Research in Memory and Cognition, 20(2), 187-197.

Kreiman, J., \& Sidtis, D. (2011). Foundations of voice studies: An interdisciplinary approach to voice production and perception. West Sussex, UK: John Wiley \& Sons.

Kreitewolf, J., Mathias, S. R., \& von Kriegstein, K. (2017). Implicit talker training improves comprehension of auditory speech in noise. Frontiers in psychology, 8, 1584.

Lavan, N., \& McGettigan, C. (2019). Toward a unified account of person perception from familiar and unfamiliar voices. PsyArXiv. 
Lavan, N., Burston, L. F., \& Garrido, L. (2019). How many voices did you hear? Natural variability disrupts identity perception from unfamiliar voices. British Journal of Psychology, 110(3), 576-593.

Lavan, N., Burston, L. F., Ladwa, P., Merriman, S. E., Knight, S., \& McGettigan, C. (2019). Breaking voice identity perception: Expressive voices are more confusable for listeners. Quarterly Journal of Experimental Psychology, 72(9), 2240-2248.

Lavan, N., Burton, A. M., Scott, S. K., \& McGettigan, C. (2019). Flexible voices: Identity perception from variable vocal signals. Psychonomic Bulletin \& Review, 26(1), 90-102.

Kreitewolf, J., Lavan, N., Kreitewolf J., Obleser, J., \& McGettigan, C. (in press2020). Familiarity and task context shape the use of acoustic information in voice identity perception. PsyArXivCognition.

Lavan, N., Merriman, S. E., Ladwa, P., Burston, L. F., Knight, S., \& McGettigan, C. (2020). 'Please sort these voice recordings into 2 identities': Effects of task instructions on performance in voice sorting studies. British Journal of Psychology, 111(3), 556569.

Lavan, N., Scott, S. K., \& McGettigan, C. (2016). Impaired generalization of speaker identity in the perception of familiar and unfamiliar voices. Journal of Experimental Psychology: General, 145(12), 1604-1614.

Mathias, S. R., \& von Kriegstein, K. (2014). How do we recognise who is speaking. Frontiers in Biosciences, 6, 92-109.

Nygaard, L. C., \& Pisoni, D. B. (1998). Talker-specific learning in speech perception. Perception \& Psychophysics, 60(3), 355-376.

Perrachione, T. K. (2017). "Speaker recognition across languages" in S. Frühholz \& P. Belin (Eds.), The Oxford Handbook of Voice Perception, Oxford: Oxford University Press.

Perrachione, T. K., Chiao, J. Y., \& Wong, P. C. (2010). Asymmetric cultural effects on perceptual expertise underlie an own-race bias for voices. Cognition, 114(1), 4255.

Stevenage, S. V., Clarke, G., \& McNeill, A. (2012). The "other-accent" effect in voice recognition. Journal of Cognitive Psychology, 24(6), 647-653.

Stevenage, S. V., Symons, A. E., Fletcher, A., \& Coen, C. (2020). Sorting through the impact of familiarity when processing vocal identity: Results from a voice sorting task. Quarterly Journal of Experimental Psychology, 73(4), 519-536.

Stringer, L., \& Iverson, P. (2019). Accent Intelligibility Differences in Noise Across Native and Nonnative Accents: Effects of Talker-Listener Pairing at AcousticPhonetic and Lexical Levels. Journal of Speech, Language, and Hearing Research, 62(7), 2213-2226.

Zarate, J. M., Tian, X., Woods, K. J., \& Poeppel, D. (2015). Multiple levels of linguistic and paralinguistic features contribute to voice recognition. Scientific reports, 5(1), 1-9. 